\title{
Early-life factors contributing to type 1 diabetes
}

\author{
Maria E. Craig ${ }^{1,2,3}$ (1) Ki Wook Kim ${ }^{1,4} \cdot$ Sonia R. Isaacs ${ }^{1,4} \cdot$ Megan A. Penno $^{5,6} \cdot$ Emma E. Hamilton-Williams $^{7}$. \\ Jennifer J. Couper ${ }^{5,6}$. William D. Rawlinson ${ }^{1,4,8}$
}

Received: 22 March 2019 / Accepted: 21 May 2019 / Published online: 27 August 2019

(C) Springer-Verlag GmbH Germany, part of Springer Nature 2019

\begin{abstract}
The incidence of type 1 diabetes has increased since the mid-twentieth century at a rate that is too rapid to be attributed to genetic predisposition alone. While the disease can occur at any age, mounting evidence from longitudinal cohort studies of at-risk children indicate that type 1 diabetes associated autoantibodies can be present from the first year of life, and that those who develop type 1 diabetes at a young age have a more aggressive form of the disease. This corroborates the hypothesis that environmental exposures in early life contribute to type 1 diabetes risk, whether related to maternal influences on the fetus during pregnancy, neonatal factors or later effects during infancy and early childhood. Studies to date show a range of environmental triggers acting at different time points, suggesting a multifactorial model of genetic and environmental factors in the pathogenesis of type 1 diabetes, which integrally involves a dialogue between the immune system and pancreatic beta cells. For example, breastfeeding may have a weak protective effect on type 1 diabetes risk, while use of an extensively hydrolysed formula does not. Additionally, exposure to being overweight pre-conception, both in utero and postnatally, is associated with increased risk of type 1 diabetes. Epidemiological, clinical and pathological studies in humans support a role for viral infections, particularly enteroviruses, in type 1 diabetes, but definitive proof is lacking. The role of the early microbiome and its perturbations in islet autoimmunity and type 1 diabetes is the subject of investigation in ongoing cohort studies. Understanding the interactions between environmental exposures and the human genome and metagenome, particularly across ethnically diverse populations, will be critical for the development of future strategies for primary prevention of type 1 diabetes.
\end{abstract}

Keywords Child · Diet · Enterovirus · Gene-environment interaction · Life course development · Microbiome $\cdot$ Pregnancy Review $\cdot$ Type 1 diabetes $\cdot$ Virus

\section{Abbreviations \\ CVB Coxsackievirus group B \\ DAISY Diabetes Autoimmunity Study in the Young}

DIPP

ENDIA Environmental Determinants of Islet Autoimmunity ER Endoplasmic reticulum
Electronic supplementary material The online version of this article (https://doi.org/10.1007/s00125-019-4942-x) contains a slideset of the figures for download, which is available to authorised users.

\section{Maria E. Craig}

m.craig@unsw.edu.au

1 School of Women's and Children's Health, University of New South Wales Faculty of Medicine, Sydney, NSW, Australia

2 Institute of Endocrinology and Diabetes, Children's Hospital at Westmead, Locked Bag 4001, Westmead, Sydney, NSW 2145, Australia

3 Discipline of Child and Adolescent Health, University of Sydney, Sydney, NSW, Australia
4 Virology Research Laboratory, Prince of Wales Hospital Randwick, Sydney, NSW, Australia

5 Robinson Research Institute, School of Paediatrics and Reproductive Health, University of Adelaide, Adelaide, SA, Australia

6 Department of Endocrinology and Diabetes, Women's and Children's Hospital, Adelaide, SA, Australia

7 University of Queensland Diamantina Institute, University of Queensland, Translational Research Institute, Brisbane, QLD, Australia

8 Serology and Virology Division, NSW Health Pathology, Prince of Wales Hospital, Sydney, NSW, Australia 
FDR First-degree relative

GRS Genetic risk score

IA Islet autoimmunity

rRNA Ribosomal RNA

SCFA Short-chain fatty acid

TEDDY The Environmental Determinants of Diabetes in the Young

TRIGR Trial to Reduce Insulin-Dependent Diabetes Mellitus in the Genetically at Risk

VIGR Viruses In the Genetically at Risk

\section{Introduction}

The accepted model that type 1 diabetes develops in an individual due to the interplay between genetic predisposition and environmental determinants has been challenged by the observation that the rising incidence of type 1 diabetes in individuals over 50 years of age is primarily associated with people who are not at increased genetic risk [1-3]. Hence, environmental determinants now appear to exert a greater influence on the risk of islet autoimmunity (IA) and type 1 diabetes than in the past. The large body of evidence to support this hypothesis is based on in vitro models, animal models, cohort studies, epidemiological analyses and in vivo observations. However, inconsistencies regarding the relative contribution of genes vs environment remain, which may be explained by the heterogeneity of type 1 diabetes across the lifespan and the globe, methodological differences in studies from which conclusions have been derived and the changing impact of factors over time. Moreover, there is a paucity of information on how the in utero milieu influences type 1 diabetes risk in the offspring. In the context of a changing permissive environment, we review the contribution of candidate early-life factors, from pregnancy through to early childhood, to the development of IA and progression to type 1 diabetes. The major cohort studies and trials referenced in this review are shown in Table 1 and factors associated with initiation of or protection from IA and progression to type 1 diabetes are summarised in the Text box.

\section{Environmental factors associated with initiation of, or protection from, IA and progression to type 1 diabetes}

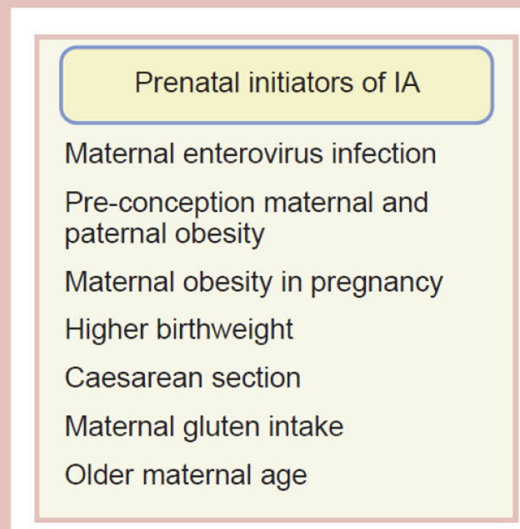

Prenatal protective factors of IA

Higher maternal vitamin D

Gestational respiratory infections
Postnatal initiators of IA

Enterovirus infection $+/-$ viral load

Respiratory infection

Infant weight gain

Early exposure to cereals, root vegetables

Greater cows' milk protein intake

Stressful life events

Postnatal protective factors of IA

Breastfeeding in early life

Early introduction of gluten or egg

Higher $n-3$ fatty acids

Probiotics

Rotavirus vaccination
Postnatal progressors to type 1 diabetes

Persistent or recurrent enterovirus infections

Accelerator hypothesis (overweight/greater height velocity)

Puberty/insulin resistance

High glycaemic load

Lower microbial diversity

Psychological stress

Postnatal protection from progression to type 1 diabetes

Limited evidence 
Table 1 Cohort studies and clinical trials investigating the relationship between early-life factors and IA and/or type 1 diabetes

\begin{tabular}{|c|c|c|c|}
\hline Cohort study/trial & Country/region & Study population & References \\
\hline Australian BABYDIAB & Australia & Birth cohort: FDR with T1D & {$[4,5]$} \\
\hline BABYDIAB & Germany & Birth cohort: offspring of parents with T1D & {$[6]$} \\
\hline BABYDIET & Germany & $\begin{array}{l}\text { RCT: at least two FDRs with T1D, or one FDR with T1D } \\
\text { and a high-risk HLA genotype }\end{array}$ & [7-9] \\
\hline DAISY & $\mathrm{CO}, \mathrm{USA}$ & Birth cohort: high-risk HLA genotypes or FDR with T1D & {$[10,11]$} \\
\hline DIABIMMUNE & $\begin{array}{l}\text { Finland, Estonia, } \\
\quad \text { Russian Karelia }\end{array}$ & Birth cohort: high-risk HLA genotypes & {$[12,13]$} \\
\hline DIPP & Finland & Birth cohort: high-risk HLA genotypes & {$[14-18]$} \\
\hline DNBC & Denmark & Birth cohort & {$[19,20]$} \\
\hline ENDIA & Australia & Pregnancy/birth cohort: FDR with T1D & {$[21,22]$} \\
\hline KVB claims data & Bavaria, Germany & Birth cohort & {$[23]$} \\
\hline MIDIA & Norway & Birth cohort: high-risk HLA genotypes & {$[24]$} \\
\hline $\mathrm{MoBa}$ & Norway & Birth cohort & {$[19,20]$} \\
\hline $\begin{array}{l}\text { National Health Insurance } \\
\text { Research database }\end{array}$ & Taiwan & Birth cohort & {$[25]$} \\
\hline Tasmanian Infant Health Survey & TAS, Australia & Birth cohort & {$[26]$} \\
\hline TEDDY & USA, Europe & Birth cohort: high-risk HLA genotypes or FDR with T1D & {$[27-33]$} \\
\hline TRIGR & $\begin{array}{l}\text { Europe, USA, } \\
\quad \text { Canada, Australia }\end{array}$ & $\begin{array}{l}\text { Primary prevention RCT from birth: FDR and high-risk HLA } \\
\text { genotypes }\end{array}$ & {$[34,35]$} \\
\hline VIGR & Australia & Birth cohort: FDR with T1D & {$[36]$} \\
\hline
\end{tabular}

DNBC, Danish National Birth Cohort; KVB, Kassenärztliche Vereinigung Bayern; MIDIA, Environmental Triggers of Type 1 Diabetes; MoBa, Norwegian Mother and Child Cohort Study; T1D, type 1 diabetes

\section{Genetics}

The risk of type 1 diabetes is tenfold higher in children with an affected first-degree relative (FDR) compared with the general population. A substantial component of this risk $(\sim 50 \%)$ can be explained by specific HLA alleles [37]. The highest risk haplotypes are $D R B 1 * 03: 01-D Q A 1 * 05: 01-D Q B 1 * 02: 01$ (known as 'DR3') and DRB1*04-DQA1*03:01$D Q B 1^{*} 03: 02$ (known as 'DR4'). In a pooled analysis of 5196 individuals with type 1 diabetes and 6359 control participants, individuals who were heterozygous for DR3/4 had an OR $>16$ for type 1 diabetes, while DR3/3 and DR4/4 homozygotes had ORs $\sim 6$ [37]. However, $<10 \%$ of individuals with HLA-conferred susceptibility develop type 1 diabetes.

Genome-wide association studies elucidated a putative role for multiple non-HLA type 1 diabetes risk loci [38]. Incorporation of multiple loci into a genetic risk score (GRS) can predict $>10 \%$ of risk for pre-symptomatic type 1 diabetes in children without an affected FDR [39]. In an ethnically diverse population from South-Eastern USA, younger age at type 1 diabetes diagnosis was associated with a higher prevalence of the DR3/4 diplotype and a higher GRS [40]. This suggests that the relative contribution of the environment may be greater in those who develop type 1 diabetes at an older age. Recently, the combination of 67 single nucleotide polymorphisms, HLA DR-DQ loci and their interactions into a revised GRS (GRS2) was highly discriminative for type 1 diabetes, particularly early-onset disease, with an area under the curve of 0.96 [41]. In the context of population screening for type 1 diabetes, which is now being undertaken through multiple programmes $[42,43]$, the GRS has substantial potential for prediction of type 1 diabetes in early life.

The application of a GRS has also provided further insights into the risk of IA and type 1 diabetes in childhood in familial vs non-familial type 1 diabetes. The Environmental Determinants of Diabetes in the Young (TEDDY) study screened infants for multiple type 1 diabetes-associated alleles and stratified genetic susceptibility into four groups based on presence of 'high-risk' DR4 allele subtypes and quartiles of GRS [27]. On comparing children with or without an FDR with type 1 diabetes, those in the highest risk group (high-risk DR4 allele subtypes and first quartile GRS) had similar rates of multiple islet autoantibodies (14.3\% vs $12.7 \%$, respectively) and type 1 diabetes (4.8\% vs $4.1 \%$, respectively). In contrast, for children in the lowest genetic susceptibility group (low-risk DR4 allele subtypes and GRS $<50$ th percentile), the risks of multiple autoantibodies in those with or without an FDR differed almost sixfold (9.2\% vs $1.6 \%$, respectively), suggesting that additional factors were enriched within families where more than one individual developed type 1 diabetes compared with the general population. The findings support the hypothesis that the contribution of genetic and environmental factors to the development of type 1 diabetes varies according to family history. 


\section{Dietary factors}

Dietary factors, including early introduction of cow's milk protein [14], overall dairy intake and early or late introduction of gluten [28], have long been implicated in the development of IA and type 1 diabetes, potentially through a mechanistic role; these dietary factors may act as antigenic triggers of autoimmunity or as co-factors in the context of gut infection and/or inflammation.

\section{Maternal diet}

The impact of maternal diet on the risk of type 1 diabetes has not been extensively assessed. In the Danish National Birth Cohort study, offspring of women with high gluten intake (>20 g/day) had double the risk of type 1 diabetes vs offspring of women with low gluten intake ( $<7 \mathrm{~g} /$ day) [44]. In the Diabetes Autoimmunity Study in the Young (DAISY), maternal intake of vitamin D via food (but not via supplements) was associated with a $63 \%$ decreased risk of IA in offspring [45]. Higher maternal vitamin D-binding protein levels and higher cord blood 25-hydroxyvitamin D [25(OH)D] was associated with a lower risk of type 1 diabetes in the offspring, with a vitamin D receptor-genotype interaction [46]. In contrast, average maternal vitamin $\mathrm{D}$ plasma concentration during pregnancy was not associated with an increased risk of type 1 diabetes in cohorts from Norway and Denmark [19]. Further data from prospective studies commencing in pregnancy, such as the Environmental Determinants of Islet Autoimmunity (ENDIA) study (anzctr.org.au registration no. ACTRN12613000794707) [21], will provide more comprehensive data on the role of perinatal vitamin D supplementation in IA and type 1 diabetes.

\section{Cow's milk protein}

DAISY demonstrated that greater intake of cow's milk protein was associated with increased IA risk in children with low/moderate risk $H L A-D R$ genotypes (adjusted HR 1.41 [95\% CI 1.08, 1.84]), but not in children with highrisk HLA-DR genotypes [10]. The Trial to Reduce InsulinDependent Diabetes Mellitus in the Genetically at Risk (TRIGR), an RCT that only recruited children with highrisk HLA genotypes, demonstrated that cow's milk ingestion during infancy did not reduce the incidence of IA [34] or type 1 diabetes [35], as compared with hydrolysed formula. In the Diabetes Prediction and Prevention (DIPP) study, exposure to cow's milk protein formula before 3 months of age was not associated with IA or type 1 diabetes, but the interaction between enterovirus infection and cow's milk exposure before the age of 3 months was associated with IA [15]. If cow's milk protein has a role in the pathogenesis of type 1 diabetes, the relationship may result from an interplay between genetic predisposition to aberrant mucosal immunity to dietary and other proteins [11], along with increased intestinal permeability ('leaky gut') and exposure to intestinal microbiota [47].

\section{Breastfeeding}

A meta-analysis of 43 studies, including 9874 cases of type 1 diabetes, demonstrated a weak protective effect of breastfeeding on type 1 diabetes risk: for exclusive breastfeeding $\geq 2$ weeks vs $<2$ weeks, the OR was $0.75(95 \%$ CI $0.64,0.88$ ) and for exclusive breastfeeding $\geq 3$ months vs $<3$ months, the OR was 0.87 (95\% CI $0.75,1.00)$. The authors concluded that their findings were difficult to interpret due to marked heterogeneity and possible biases, particularly recall bias [48]. For ethical reasons, it will not be possible to address the question of exclusive breastfeeding in an RCT; however breastfeeding should be encouraged in children at risk of type 1 diabetes, as for the general population, due to other established benefits [49].

\section{Gluten}

Both early and delayed introduction of gluten have been implicated in the risk of IA and type 1 diabetes $[6,28]$. In TEDDY (which included $\sim 700$ children with IA), early introduction of gluten before the age of 4 months reduced IA risk (adjusted HR 0.67 [95\% CI 0.54, 0.98]), while late introduction of gluten (after 9 months of age) was not associated with increased IA risk [28]. Similarly, the BABYDIET study showed that delaying gluten exposure until 12 months of age in at-risk children was not associated with reduced IA risk [7]. The modest HRs in TEDDY and small sample size in BABYDIET suggest that further investigation into the effects of timing of gluten introduction on IA/type 1 diabetes risk is warranted in other cohorts, as well as studies into the interaction of gluten intake with other IA/type 1 diabetes risk variables, such as breastfeeding and infection.

\section{Specific foods and micro- and macro-nutrients}

Additional dietary factors that have been variably associated with risk of IA and type 1 diabetes, such as root vegetables $[16,17]$ or berries and fruit [16], were not shown to have significant associations with these conditions in the TEDDY dietary analyses [28]. Only the introduction of egg before 9 months of age was associated with a reduced IA risk (adjusted HR 0.8 [95\% CI $0.72,0.99] ; p=0.035$ ). While the number of individuals in TEDDY [28] is much larger than the earlier studies in this area (highlighting the potential for small-study effects [50]), the discrepant findings between these studies may be related to chance findings in any of the studies, particularly given the large number of dietary variables measured and the lack of adjustment for multiple 
comparisons $[16,28]$. Other possible explanations include geographic differences in study populations, methodological differences between studies, changes in dietary practices over time and interaction between diet and other associated biological systems, such as the metabolome and gut microbiome.

\section{Overweight/obesity}

The rising incidence of type 1 diabetes largely parallels population rates of overweight/obesity over time. Using combined data from two large birth cohort studies in Norway and Denmark, type 1 diabetes risk in the offspring was modestly associated with maternal pre-pregnancy obesity (adjusted HR 1.41) and paternal obesity (adjusted HR 1.51) [20]. A metaanalysis of 29 predominantly European studies demonstrated that birthweight $>3.5 \mathrm{~kg}$ was associated with an increased risk of type 1 diabetes, after adjustment for potential confounders [51]. In the Australian BABYDIAB study, weight and BMI $z$ scores during infancy were continuous predictors of IA risk, with a weight $z$ score $>0$ conferring a more than twofold risk of IA (HR 2.6) [4]. The effect of exposure to being overweight pre-conception, in utero and postnatally may be mediated by accompanying insulin resistance $[52,53]$.

\section{Infections}

An infectious aetiology for type 1 diabetes has been postulated for almost a century [54]. Multiple prospective cohort studies have investigated the role of early-life infections in type 1 diabetes, using prospective clinical data, as well as specific microbial and virological testing [23-26, 29, 55]. Recently, our understanding of the role of infections has been enhanced by the use of 'omics' technologies to determine the relationship between the microbiome (including bacteria, bacteriophages and viruses), transcriptome and proteome in the development of IA/type 1 diabetes.

\section{Maternal virus infection}

Pregnancy represents a complex immunological state in which bias towards helper $\mathrm{T}$ lymphocyte-associated cytokines (Th2 bias) diminishes cell-mediated immunity and increases vulnerability to intracellular infections, including viruses [56, 57]. Acute perinatal infections, such as rubella and infection with cytomegalovirus, can cause significant perinatal morbidity, with resulting clinical syndromes including diabetes; although most cases of congenital rubella syndrome have an atypical form of diabetes without evidence of IA [58]. In our systematic review and metaanalysis of 2039 mothers and 953 offspring, we found a significant association between virus infection during pregnancy and childhood type 1 diabetes (OR 2.2) but not IA [55]. The TEDDY study showed that retrospectively reported maternal respiratory infections during pregnancy were associated with a reduced risk of developing insulin autoantibodies in children with the CTLA4 G allele polymorphism (HR 0.64 [95\% CI 0.45, 0.91]), suggesting a protective role for this allele [30]. Our systematic review highlighted the need for data from prospective cohorts commencing in pregnancy, with frequently obtained clinical samples to document infection, along with pathogenesis studies, to establish an aetiological link between in utero infections and type 1 diabetes in the offspring. The ongoing ENDIA study will address these questions, with a strong focus on 'omics' [21].

\section{Childhood infections}

Infections in early life increase the risk of IA and type 1 diabetes in at-risk and unselected population cohorts [23, 24, 26, 29]. In TEDDY, prospectively collected parent-reported history of recent respiratory infections in children before 4 years of age was associated with a modestly increased $(5.6 \%)$ IA risk. The incidence of both IA and respiratory infections peaked at 6 9 months of age (Fig. 1) and a higher rate of respiratory infections was observed in the 9 months prior to seroconversion to IA [29]. In a population-based cohort from Germany, type 1 diabetes risk was significantly increased in children who experienced respiratory tract infections before 6 months of age [23]. Similarly, in an Australian birth cohort study of healthy children, type 1 diabetes risk was increased in association with an early upper respiratory tract infection before the age of 5 weeks (adjusted OR 2.7) or ear infection by the age of 12 weeks (adjusted OR 3.4) [26]. Since these studies were based on parental

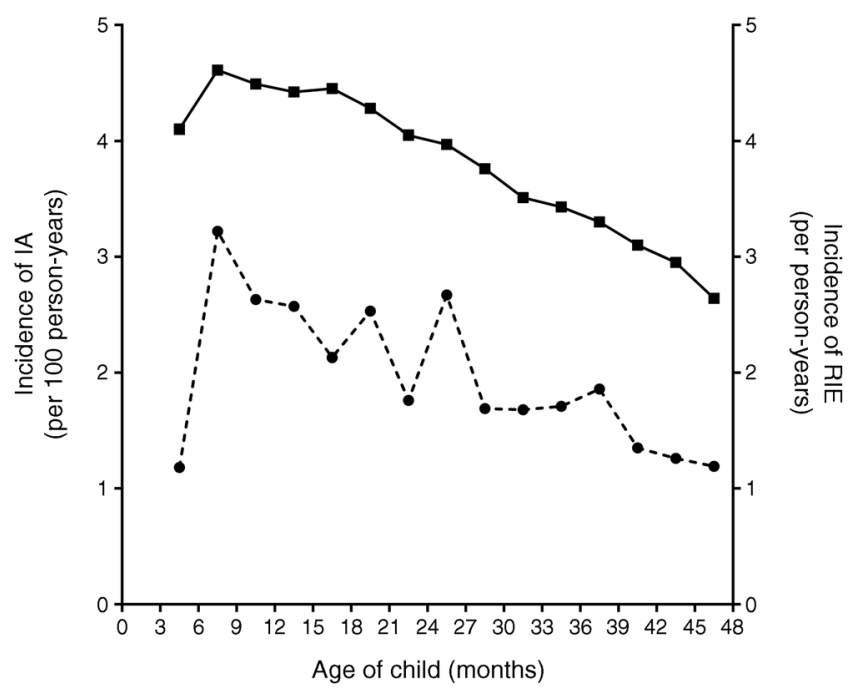

Fig. 1 Incidence of IA per 100 person-years (dashed line) in TEDDY peaked between 6 and 9 months of age and declined thereafter. Incidence of respiratory infectious episode (RIE) per person-years (solid line) also peaked between 6 and 9 months and declined thereafter. Age-specific RIE rates were strongly correlated with the decline in IA incidence (Spearman's correlation $=0.71 ; p=0.003$ ). Adapted from [29] by permission from Springer Nature. This figure is available as part of a downloadable slideset 
reporting of infection, causative agents were not identified. In contrast, a large population-based study of $>1$ million cases and controls from Taiwan demonstrated that enterovirus infection before 18 years of age was associated with an increased risk of subsequent type 1 diabetes (adjusted HR 1.5) [25].

Such studies cannot determine whether the associations between early infections and IA or type 1 diabetes reflect increased exposure to viruses or an underlying susceptibility to virus infection due to a dysregulated immune response [59]. This may have a genetic basis, as antiviral response genes, such as IFIHI CD69, and IL2RA, are among the loci linked to type 1 diabetes susceptibility [60]. In support of this hypothesis, a type I interferon-inducible transcriptional signature was increased in the peripheral blood of children from the BABYDIET study before seroconversion to IA and correlated with recent self-reported respiratory infections [8]. Also, it is possible that treatment with antibiotics in early life [61], which alter the gut microbiome, may explain the relationship between infection and IA/type 1 diabetes; although, antibiotic treatment likely serves as a confounder. Infections may contribute to IA and type 1 diabetes in young children through activation of interferon signalling pathways and pattern recognition receptors following bacterial, viral and, possibly, other infections [59]. This may result in beta cell inflammation, activation of autoreactive and bystander $\mathrm{CD} 8^{+} \mathrm{T}$ cells and progressive autoimmune-mediated beta cell destruction. The detection of IFN- $\alpha$ and increased HLA class I expression in beta cells of individuals with type 1 diabetes is consistent with this model [62].

\section{Viruses}

Numerous clinical, epidemiological and experimental studies have reported associations between IA or type 1 diabetes and viruses, particularly enteroviruses [63], but also herpesviruses (cytomegalovirus, Epstein-Barr virus) [64, 65], rotavirus [5], rubivirus (rubella) [66], rubulavirus (mumps) [66] and parechoviruses [67] during infancy and childhood. Potential mechanisms by which viruses interact with genetic predisposition to induce an autoimmune assault on pancreatic beta cells have been comprehensively reviewed elsewhere $[59,63]$.

Enteroviruses In our systematic review and meta-analysis of 26 studies involving over 4400 participants, we found a significant association between enterovirus infection, IA and type 1 diabetes; for newly diagnosed type 1 diabetes, the OR was 12.7 (95\% CI 6.4, 25.0) [68]. There was significant heterogeneity by geographical region, but we could not stratify the analysis by enterovirus genotype because the majority of studies did not provide this information.

DIPP demonstrated a temporal association between the appearance of the first diabetes-associated autoantibodies and enterovirus infection, based on both serology and PCR for enterovirus genes [69-71]. In particular, neutralising antibodies to coxsackievirus group B (CVB)1 increased the risk of IA, which was attenuated by the presence of maternal antibodies to the virus. In contrast, antibodies to CVB3 and CVB6 were associated with lower IA risk, which may be due to immunological cross protection against CVB1. These associations were also present in children who progressed to type 1 diabetes [70].

Rotaviruses Recent reports from Australia and the USA have demonstrated a reduced incidence of type 1 diabetes in young children following the introduction of rotavirus vaccination $[72,73]$.

Virome Although strong evidence supports a predominant role for enteroviruses in the pathogenesis of type 1 diabetes [68], the use of targeted detection methods (PCR, serology, etc.) raises significant concern for investigation bias towards enteroviruses. With the advent of high-throughput sequencing technologies, it is now possible to screen for all viruses (the 'virome') simultaneously, eliminating such bias. However, early application of virome sequencing in TEDDY [31] and DIPP [18] proved difficult due to the limited sensitivity caused by the overwhelming background of non-viral nucleic acid in clinical specimens [74].

To significantly improve the sensitivity of virome detection by up to 10,000-fold [74], we and others applied a novel viral enrichment strategy (VirCapSeq-VERT) to characterise the virome of children with IA in the Viruses In the Genetically at Risk (VIGR) study [36] and BABYDIET [9]. The sensitivity and specificity for virus detection using this method is on par with targeted PCR, unlike other virome-sequencing methods that use physical enrichment, which are less sensitive $[36,74]$. In our analysis of the gut and plasma virome of 45 individuals with IA and 48 matched control participants, we detected 28 genera of viruses and $62 \%$ of children were positive for $\geq 1$ vertebrate-infecting virus [36]. This represents a more than threefold higher positivity than previously reported in the DIPP virome analysis [18]. We identified 129 viruses as differentially abundant in the gut of individuals with IA vs control participants, including five enterovirus A genotypes, which were significantly more abundant in those with IA. While the sample size is small, these findings further support the contribution of enteroviruses to the development of IA and corroborate the proposal that viral load may influence IA risk [36].

In our analysis of the longitudinal gut virome of 61 pregnant women (35 with type 1 diabetes and 26 without) in ENDIA, using VirCapSeq-VERT [22], 63\% of samples tested positive for at least one virus and 29 genera of eukaryotic viruses were detected (Fig. 2). Moreover, there was more than a twofold difference in the abundance of 77 viruses between the two maternal groups, including eight enterovirus B types, which were present at a higher abundance in women with type 

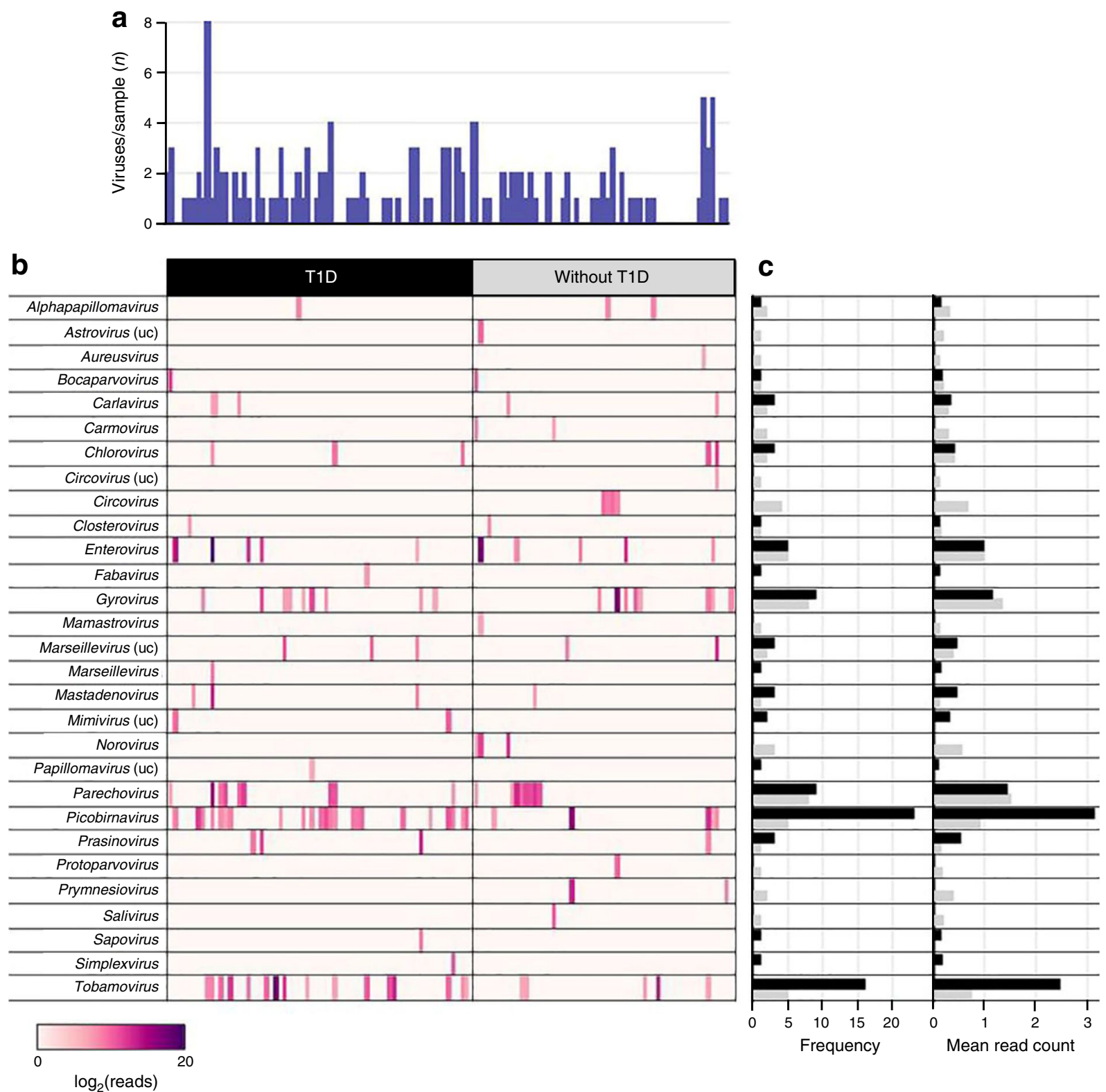

Fig. 2 Eukaryotic gut virome of 61 women during pregnancy in the ENDIA study. Viruses were detected using VirCapSeq-VERT. (a) The number of viruses detected per specimen. Each bar represents one specimen. (b) A heatmap of viral reads ( $\log _{2}$ scale) sequenced in 124 faecal samples collected from 35 women with type 1 diabetes ( $n=69$ samples) and 26 without type 1 diabetes ( $n=55$ samples) during pregnancy is shown. Only viruses with $\geq 100$ reads matched using the Basic Local

1 diabetes. These findings provide novel insight into the diversity and dynamics of the gut virome during pregnancy and demonstrate a distinct profile of viruses during pregnancy in women with type 1 diabetes. It will be important to determine whether these differences remain significant in the full ENDIA cohort, and whether they translate to altered risk for IA and type 1 diabetes in the offspring. These findings may be informative for prevention strategies, such as enterovirus vaccination [63].
Alignment Search Tool (BLAST) at the species level were included and represented at the genus level. (c) The frequency of each virus within the case or control group and the mean log read counts. Black bars, women with type 1 diabetes; grey bars, women without type 1 diabetes. T1D, type 1 diabetes; uc, unclassified. Reproduced from [22], by permission of Oxford University Press. This figure is available as part of a downloadable slideset

Phageome Gut bacteriophages are relatively unexplored in the pathogenesis of type 1 diabetes. Compared with eukaryotic viruses, they are far more ubiquitous, constituting the vast majority of viral sequences in the gut [18]. Analysis of the gut phageome in children with IA in the DIABIMMUNE study revealed significantly lower richness and Shannon diversity of bacteriophages in individuals with IA vs matched control participants [12]. Furthermore, a subset of bacteriophage sequences was directly or inversely associated with 
seroconversion to IA and highly predictive for discriminating between cases and controls. Interestingly, these differences in bacteriophage populations before seroconversion were observed despite the lack of distinguishing bacterial signatures from the same samples [75]. This suggests that changes in the phageome prior to seroconversion may contribute to changes in bacterial diversity after seroconversion.

\section{Bacterial microbiome}

Dysbiosis of the gut microbiota has been associated with IA and type 1 diabetes, yet the functional consequences to the host of this dysbiosis are still being unravelled. The composition of the gut microbiome is strongly affected by the maternal environment, diet (particularly breastfeeding in early life) and use of antibiotics and probiotics. The microbiome interacts closely with the host immune system, making it a strong contender for providing a mechanistic link between multiple environmental modifiers and IA.

\section{Maternal microbiome}

A landmark study of the gut microbiome in healthy human pregnancy showed marked changes from the first to the third trimester, to a more proinflammatory microbiome [76]. Such changes could be considered adaptive to support the growing fetus and weight gain of pregnancy; however, these changes were not replicated in subsequent studies of pregnancy, with reports of a stable gestational microbiome across different body sites [77]. Maternal and, potentially, paternal factors influence the infant's microbiome after rapid changes during the first year of life. Maternal weight, pregnancy weight gain and complications of pregnancy, such as diabetes and pre-eclampsia, all influence the composition and functional capacity of the gut microbiome, as followed through preschool years [32]. Antibiotic use in pregnancy can affect the maternal and infant microbiome, but overall exposure, number of courses or antibiotic type (narrow vs broad) in utero were not associated with type 1 diabetes risk [78].

\section{Infant microbiome}

The gut microbiome has a critical role in the early development of immune regulation in the infant [79]. The early microbiome, and its perturbations in association with IA and type 1 diabetes, is the subject of investigation within multiple cohorts. Whilst most studies used $16 \mathrm{~S}$ ribosomal RNA (rRNA) sequencing to assess community composition, modern metagenomic methods more accurately identify functional and strain-specific differences in the microbiome [13] and other approaches, such as faecal metaproteomics, can identify host-microbiota interactions [80].
Microbial composition during early life is influenced by mode of birth, breastfeeding, introduction of formula milk and solid foods, antibiotic and probiotic use, and bacterial and viral infections. A decrease in diversity and stability of case microbiomes over time, as well as a reduction in shortchain fatty acid (SCFA) production and gut integrityassociated bacterial genes, were associated with IA and type 1 diabetes $[33,75]$. In DIABIMMUNE, alpha diversity decreased after seroconversion in individuals who progressed from IA to type 1 diabetes, with the onset of type 1 diabetes being preceded by inflammation-associated microbes and functional pathways [75].

The recent longitudinal analysis of stool microbiomes in TEDDY (mainly white non-Hispanic children), using $16 \mathrm{~S}$ rRNA and metagenomic sequencing, is the largest study to examine the infant microbiome $[32,33]$. Three phases were described: (1) a developmental phase (3-14 months); (2) a transitional phase (15-30 months); and (3) a stable phase (31-46 months). Partial or exclusive breastfeeding was the most significant factor associated with microbial composition, resulting in increased Bifidobacterium species. Weaning led to faster maturation of the gut microbiome, driven by the breast milk cessation, heralding the appearance of bacteria belonging to the phylum Firmicutes. Other factors that significantly associated, but to a lesser degree, with infant microbiota composition were geographical location, antibiotic use, probiotic use, household siblings, furry pets and vitamin D supplementation [32].

Further, breast milk feeding between 3 and 14 months was solely associated with bacterial metabolic potential. Pathways involving metabolic functions associated with the adult microbiome were increased in non-breastfed infants up to 14 months of age [32]. A reduction in gene families involved in the degradation of human milk oligosaccharides to SCFA and an increase in mucin-degrading genes after weaning indicated a strain-shift, whereby new microbiota, including Enterococcus species, were increasingly responsible for these functions [78].

Relatively modest alterations in the microbial composition were associated with IA or type 1 diabetes in both the amplicon sequencing [32] and metagenomic sequencing [33] arms of TEDDY. Control participants had higher levels of Lactococcus and Streptococcus, both of which are common in dairy products. Gut microbiomes of children in the TEDDY study who did or did not develop IA or type 1 diabetes, therefore, did not reveal clear taxonomic differences and there was considerable geographical heterogeneity across the study sites. However, importantly, on metagenomic analysis, the microbiomes of progressors to IA or type 1 diabetes contained significantly higher numbers of genes involved in pathways involved in fermentation and production of SCFA byproducts. SCFA bacterial products regulate host metabolism and immunity. Butyrate, in particular, maintains gut epithelial integrity, 
supports differentiation of regulatory $\mathrm{T}$ cells and promotes anti-inflammatory responses, and both butyrate and acetate have been shown to prevent diabetes in the NOD mouse. The TEDDY study findings, therefore, extend experimental data in the NOD mouse [81] and smaller human studies [82] that support a protective effect of SCFA on the development of type 1 diabetes. Interestingly, there was geographical variability as to which SCFA pathways (acetate, butyrate or propionate) were altered in children with IA/type 1 diabetes in the TEDDY study, and the taxa with which these were associated. This suggests that changes in the overall molecular functions of the microbiota may be more uniformly associated with type 1 diabetes and IA, rather than specific taxa. A recent study utilising metaproteomic analysis of stool samples sheds light on the functional impact of altered microbiota on the host; the abundance of proteins from the mucous barrier and epithelial adhesion molecules were positively associated with taxa that were abundant in control participants compared with individuals with IA or type 1 diabetes [80]. These findings further support gut epithelial integrity as a key characteristic of the microbiome in healthy individuals.

Despite TEDDY's large sample size, most associations were modest in effect size, which may be attributed to differing type 1 diabetes endotypes, high geographical heterogeneity and a lack of samples from early life (prior to 3 months of age) [33]. The impact of the microbiome and its products may be different with different genetic risk alleles and in different disease phenotypes. The role of other co-inhabitants, such as the mycobiome, is unknown, as is the impact of the dramatic changes in biological systems before and after birth. Studies investigating pregnancy to early-life cohorts, such as ENDIA [21] and DIPP Novum, will address these unknowns.

\section{Crosstalk between early-life factors and pancreatic beta cells}

While a diverse spectrum of early-life factors putatively influence the development of IA and type 1 diabetes, the mechanisms underlying their contribution to the autoimmune assault that specifically targets pancreatic beta cells are yet to be fully elucidated [59]. One potential route is the induction of stress in the endoplasmic reticulum (ER), where the synthesis/folding of proinsulin takes place [83]. During insulitis, proinflammatory cytokines released by infiltrating immune cells disrupt ER homeostasis, triggering the unfolded protein response. This adaptive phase is believed to play a critical role in the development of IA by promoting the generation of neoantigens and activating inflammatory responses [84]. Furthermore, ER stress induces translational errors, giving rise to defective ribosomal products of insulin that are highly immunogenic and targeted by $\mathrm{T}$ cell autoreactivity in type 1 diabetes [85].

\section{Research gaps and future directions}

The discrepant findings from studies investigating the multitude of putative aetiological determinants of IA and type 1 diabetes, with largely modest associations observed, highlight the importance of adequately powered studies, consistent definitions of exposure and outcome measures, multifactorial modelling of pathogenesis and meta-analyses, which can also explore publication bias and study heterogeneity. Findings from small or pilot studies may be chance associations, particularly when not replicated in larger cohorts or trials, as may be the case for TRIGR [35]. However, large studies and 'big data' are not immune from bias or chance findings [86]. Key to unravelling the heterogeneous nature of type 1 diabetes is the exploration of genetic susceptibility and validation of GRSs in diverse populations. Studies need to take account of the interplay between development of islet autoimmunity and/or type 1 diabetes and environmental factors, stratified by genetic, ethnic and global variation, particularly in early life. While incidence rates for type 1 diabetes are highest in Scandinavia/Northern Europe [87], the caseload of type 1 diabetes globally includes regions that have not been comprehensively studied, such as the Middle East, Africa, Asia and South America. For interventions aimed at primary, secondary and tertiary prevention of type 1 diabetes to be successful, understanding the heterogeneity of type 1 diabetes is essential. In the era of 'omics', the future potential to elucidate the interaction between multiple biological systems in IA/type 1 diabetes is immense.

Funding MEC is supported by an Australian National Health and Medical Research Council (NHMRC) Practitioner fellowship (APP1045777) and a JDRF International grant (3-SRA-2017-417-A-N). SRI is supported by an Australian Government Research Training Program Scholarship. EEH-W is supported by grants from JDRF (3SRA-2019-730-S-B, 2-SRA-2019-703-M-B) and a Children's Hospital Foundation grant (WIS0202018). The ENDIA Study is supported by JDRF Australia, the recipient of the Australian Research Council Special Research Initiative in Type 1 Juvenile Diabetes, The Leona M. and Harry B. Helmsley Charitable Trust, JDRF International and the JDRF/NHMRC Centre of Research Excellence for the Protection of Pancreatic Beta Cells (APP1078106; JJC, MEC, MP, WDR).

Duality of interests The authors declare that there is no duality of interest associated with this manuscript.

Contribution statement All authors contributed to the conception and design, interpretation and critical appraisal of data, drafting of the review and revising it critically for important intellectual content. All authors approved of the version to be published.

\section{References}

1. Hermann R, Knip M, Veijola R et al (2003) Temporal changes in the frequencies of HLA genotypes in patients with type 1 diabetesindication of an increased environmental pressure? Diabetologia 46(3):420-425. https://doi.org/10.1007/s00125-003-1045-4 
2. Gillespie KM, Bain SC, Barnett AH et al (2004) The rising incidence of childhood type 1 diabetes and reduced contribution of high-risk HLA haplotypes. Lancet 364(9446):1699-1700. https:// doi.org/10.1016/S0140-6736(04)17357-1

3. Fourlanos S, Varney MD, Tait BD et al (2008) The rising incidence of type 1 diabetes is accounted for by cases with lower-risk human leukocyte antigen genotypes. Diabetes Care 31(8):1546-1549. https://doi.org/10.2337/dc08-0239

4. Couper JJ, Beresford S, Hirte C et al (2009) Weight gain in early life predicts risk of islet autoimmunity in children with a first-degree relative with type 1 diabetes. Diabetes Care 32(1):94-99. https:// doi.org/10.2337/dc08-0821

5. Honeyman MC, Coulson BS, Stone NL et al (2000) Association between rotavirus infection and pancreatic islet autoimmunity in children at risk of developing type 1 diabetes. Diabetes 49(8): 1319-1324. https://doi.org/10.2337/diabetes.49.8.1319

6. Ziegler AG, Schmid S, Huber D, Hummel M, Bonifacio E (2003) Early infant feeding and risk of developing type 1 diabetesassociated autoantibodies. JAMA 290(13):1721-1728. https://doi. org/10.1001/jama.290.13.1721

7. Hummel S, Pfluger M, Hummel M, Bonifacio E, Ziegler AG (2011) Primary dietary intervention study to reduce the risk of islet autoimmunity in children at increased risk for type 1 diabetes: the BABYDIET study. Diabetes Care 34(6):1301-1305. https://doi. org $10.2337 / \mathrm{dc} 10-2456$

8. Ferreira RC, Guo H, Coulson RM et al (2014) A type I interferon transcriptional signature precedes autoimmunity in children genetically at risk for type 1 diabetes. Diabetes 63(7):2538-2550. https:// doi.org/10.2337/db13-1777

9. Hippich M, Oleynik A, Jain K et al (2018) Searching peripheral blood mononuclear cells of children with viral respiratory tract infections preceding islet autoimmunity for viruses by highthroughput sequencing. Acta Diabetol 55(8):881-884. https://doi. org/10.1007/s00592-018-1138-7

10. Lamb MM, Miller M, Seifert JA et al (2015) The effect of childhood cow's milk intake and HLA-DR genotype on risk of islet autoimmunity and type 1 diabetes: the Diabetes Autoimmunity Study in the Young. Pediatr Diabetes 16(1):31-38. https://doi.org/ 10.1111/pedi. 12115

11. Harrison LC, Honeyman MC (1999) Cow's milk and type 1 diabetes: the real debate is about mucosal immune function. Diabetes 48(8):1501-1507. https://doi.org/10.2337/diabetes.48.8.1501

12. Zhao G, Vatanen $T$, Droit L et al (2017) Intestinal virome changes precede autoimmunity in type I diabetes-susceptible children. Proc Natl Acad Sci U S A 114(30):E6166-E6175. https://doi.org/10. 1073/pnas.1706359114

13. Vatanen T, Plichta DR, Somani J et al (2019) Genomic variation and strain-specific functional adaptation in the human gut microbiome during early life. Nat Microbiol 4(3):470-479. https://doi.org/10.1038/s41564-018-0321-5

14. Virtanen SM, Rasanen L, Ylonen K et al (1993) Early introduction of dairy products associated with increased risk of IDDM in Finnish children. The Childhood in Diabetes in Finland Study Group. Diabetes 42(12):1786-1790. https://doi.org/10.2337/diab.42.12. 1786

15. Lempainen J, Tauriainen $\mathrm{S}$, Vaarala $\mathrm{O}$ et al (2012) Interaction of enterovirus infection and cow's milk-based formula nutrition in type 1 diabetes-associated autoimmunity. Diabetes Metab Res Rev 28(2):177-185. https://doi.org/10.1002/dmrr.1294

16. Virtanen SM, Kenward MG, Erkkola M et al (2006) Age at introduction of new foods and advanced beta cell autoimmunity in young children with HLA-conferred susceptibility to type 1 diabetes. Diabetologia 49(7):1512-1521. https://doi.org/10.1007/ s00125-006-0236-1

17. Virtanen SM, Takkinen HM, Nevalainen J et al (2011) Early introduction of root vegetables in infancy associated with advanced ss- cell autoimmunity in young children with human leukocyte antigen-conferred susceptibility to type 1 diabetes. Diabet Med 28(8):965-971. https://doi.org/10.1111/j.1464-5491.2011.03294.x

18. Kramna L, Kolarova K, Oikarinen S et al (2015) Gut virome sequencing in children with early islet autoimmunity. Diabetes Care 38(5):930-933. https://doi.org/10.2337/dc14-2490

19. Thorsen SU, Marild K, Olsen SF et al (2018) Lack of association between maternal or neonatal vitamin $\mathrm{D}$ status and risk of childhood type 1 diabetes: a Scandinavian case-cohort study. Am J Epidemiol 187(6):1174-1181. https://doi.org/10.1093/aje/kwx361

20. Magnus MC, Olsen SF, Granstrom C et al (2018) Paternal and maternal obesity but not gestational weight gain is associated with type 1 diabetes. Int J Epidemiol 47(2):417-426. https://doi.org/10. 1093/ije/dyx266

21. Penno MA, Couper JJ, Craig ME et al (2013) Environmental determinants of islet autoimmunity (ENDIA): a pregnancy to early life cohort study in children at-risk of type 1 diabetes. BMC Pediatr 13(1):124. https://doi.org/10.1186/1471-2431-13-124

22. Wook Kim K, Allen DW, Briese T et al (2019) Distinct gut virome profile of pregnant women with type 1 diabetes in the ENDIA study. Open Forum Infect Dis 6(2):ofz025. https://doi.org/10. 1093/ofid/ofz025

23. Beyerlein A, Donnachie E, Jergens S, Ziegler AG (2016) Infections in early life and development of type 1 diabetes. JAMA 315(17): 1899-1901. https://doi.org/10.1001/jama.2016.2181

24. Rasmussen T, Witso E, Tapia G, Stene LC, Ronningen KS (2011) Self-reported lower respiratory tract infections and development of islet autoimmunity in children with the type 1 diabetes high-risk HLA genotype: the MIDIA study. Diabetes Metab Res Rev 27(8): 834-837. https://doi.org/10.1002/dmrr. 1258

25. Lin HC, Wang CH, Tsai FJ et al (2015) Enterovirus infection is associated with an increased risk of childhood type 1 diabetes in Taiwan: a nationwide population-based cohort study. Diabetologia 58(1):79-86. https://doi.org/10.1007/s00125-014-3400-z

26. Ponsonby AL, Pezic A, Cochrane J et al (2011) Infant anthropometry, early life infection, and subsequent risk of type 1 diabetes mellitus: a prospective birth cohort study. Pediatr Diabetes 12(4 Pt 1):313-321. https://doi.org/10.1111/j.1399-5448.2010.00693.x

27. Hippich M, Beyerlein A, Hagopian WA et al (2019) Genetic contribution to the divergence in type 1 diabetes risk between children from the general population and children from affected families. Diabetes 68(4):847-857. https://doi.org/10.2337/db18-0882

28. Uusitalo U, Lee HS, Andren Aronsson C et al (2018) Early infant diet and islet autoimmunity in the TEDDY study. Diabetes Care 41(3):522-530. https://doi.org/10.2337/dc17-1983

29. Lonnrot M, Lynch KF, Elding Larsson H et al (2017) Respiratory infections are temporally associated with initiation of type 1 diabetes autoimmunity: the TEDDY study. Diabetologia 60(10):19311940. https://doi.org/10.1007/s00125-017-4365-5

30. Lynch KF, Lee HS, Torn C et al (2018) Gestational respiratory infections interacting with offspring HLA and CTLA-4 modifies incident beta-cell autoantibodies. J Autoimmun 86:93-103. https://doi.org/10.1016/j.jaut.2017.09.005

31. Lee HS, Briese T, Winkler C et al (2013) Next-generation sequencing for viruses in children with rapid-onset type 1 diabetes. Diabetologia 56(8):1705-1711. https://doi.org/10.1007/s00125013-2924-y

32. Stewart CJ, Ajami NJ, O'Brien JL et al (2018) Temporal development of the gut microbiome in early childhood from the TEDDY study. Nature 562(7728):583-588. https://doi.org/10.1038/s41586018-0617-x

33. Vatanen T, Franzosa EA, Schwager R et al (2018) The human gut microbiome in early-onset type 1 diabetes from the TEDDY study. Nature 562(7728):589-594. https://doi.org/10.1038/s41586-018$0620-2$ 
34. Knip M, Akerblom HK, Becker D et al (2014) Hydrolyzed infant formula and early $\beta$-cell autoimmunity: a randomized clinical trial. JAMA 311(22):2279-2287. https://doi.org/10.1001/jama.2014. 5610

35. Writing Group for the TRIGR Study Group (2018) Effect of hydrolyzed infant formula vs conventional formula on risk of type 1 diabetes: the TRIGR randomized clinical trial. JAMA 319(1):3848. https://doi.org/10.1001/jama.2017.19826

36. Kim KW, Horton JL, Pang CNI et al (2019) Higher abundance of enterovirus A species in the gut of children with islet autoimmunity. Sci Rep 9(1):1749. https://doi.org/10.1038/s41598-018-38368-8

37. Noble JA, Valdes AM (2011) Genetics of the HLA region in the prediction of type 1 diabetes. Curr Diab Rep 11(6):533-542. https:// doi.org/10.1007/s11892-011-0223-x

38. Rich SS, Akolkar B, Concannon P et al (2009) Overview of the Type I Diabetes Genetics Consortium. Genes Immun 10(Suppl 1): S1-S4. https://doi.org/10.1038/gene.2009.84

39. Bonifacio E, Beyerlein A, Hippich M et al (2018) Genetic scores to stratify risk of developing multiple islet autoantibodies and type 1 diabetes: a prospective study in children. PLoS Med 15(4): e1002548. https://doi.org/10.1371/journal.pmed.1002548

40. Perry DJ, Wasserfall CH, Oram RA et al (2018) Application of a genetic risk score to racially diverse type 1 diabetes populations demonstrates the need for diversity in risk-modeling. Sci Rep 8(1):4529. https://doi.org/10.1038/s41598-018-22574-5

41. Sharp SA, Rich SS, Wood AR et al (2019) Development and standardization of an improved type 1 diabetes genetic risk score for use in newborn screening and incident diagnosis. Diabetes Care 42(2): 200-207. https://doi.org/10.2337/dc18-1785

42. Hommel A, Haupt F, Delivani P et al (2018) Screening for type 1 diabetes risk in newborns: the Frederlk Pilot Study in Saxony. Horm Metab Res 50(1):44-49. https://doi.org/10.1055/s-0043120921

43. Zhao Z, Miao D, Michels A et al (2016) A multiplex assay combining insulin, GAD, IA-2 and transglutaminase autoantibodies to facilitate screening for pre-type 1 diabetes and celiac disease. $\mathrm{J}$ Immunol Methods 430:28-32. https://doi.org/10.1016/j.jim.2016. 01.011

44. Antvorskov JC, Halldorsson TI, Josefsen K et al (2018) Association between maternal gluten intake and type 1 diabetes in offspring: national prospective cohort study in Denmark. BMJ 362:k3547. https://doi.org/10.1136/bmj.k3547

45. Fronczak CM, Baron AE, Chase HP et al (2003) In utero dietary exposures and risk of islet autoimmunity in children. Diabetes Care 26(12):3237-3242. https://doi.org/10.2337/diacare.26.12.3237

46. Tapia G, Marild K, Dahl SR et al (2019) Maternal and newborn vitamin d-binding protein, vitamin D levels, vitamin $\mathrm{D}$ receptor genotype, and childhood type 1 diabetes. Diabetes Care 42(4): 553-559. https://doi.org/10.2337/dc18-2176

47. Li X, Atkinson MA (2015) The role for gut permeability in the pathogenesis of type 1 diabetes - a solid or leaky concept? Pediatr Diabetes 16(7):485-492. https://doi.org/10.1111/pedi. 12305

48. Cardwell CR, Stene LC, Ludvigsson J et al (2012) Breast-feeding and childhood-onset type 1 diabetes: a pooled analysis of individual participant data from 43 observational studies. Diabetes Care 35(11):2215-2225. https://doi.org/10.2337/dc12-0438

49. Stordal K, Lundeby KM, Brantsaeter AL et al (2017) Breastfeeding and infant hospitalization for infections: large cohort and sibling analysis. J Pediatr Gastroenterol Nutr 65(2):225-231. https://doi.org/10.1097/MPG.0000000000001539

50. Sterne JA, Gavaghan D, Egger M (2000) Publication and related bias in meta-analysis: power of statistical tests and prevalence in the literature. J Clin Epidemiol 53(11):1119-1129. https://doi.org/10. 1016/S0895-4356(00)00242-0
51. Cardwell CR, Stene LC, Joner G et al (2010) Birthweight and the risk of childhood-onset type 1 diabetes: a meta-analysis of observational studies using individual patient data. Diabetologia 53(4): 641-651. https://doi.org/10.1007/s00125-009-1648-5

52. Fourlanos $\mathrm{S}$ (2005) Insulin resistance in children and adolescents with type 1 diabetes mellitus: relation to obesity. Pediatr Diabetes 6(1):3-4. https://doi.org/10.1111/j.1399-543X.2005.00094.x

53. Islam ST, Srinivasan S, Craig ME (2014) Environmental determinants of type 1 diabetes: a role for overweight and insulin resistance. J Paediatr Child Health 50(11):874-879. https://doi.org/10. 1111/jpc. 12616

54. Gundersen E (1927) Is diabetes of infectious origin? J Infect Dis 41(3):197-202. https://doi.org/10.1093/infdis/41.3.197

55. Allen DW, Kim K, Rawlinson WD, Craig ME (2018) Maternal virus infections in pregnancy and type 1 diabetes in their offspring: systematic review and meta-analysis of observational studies. Rev Med Virol 28(3):e1974. https://doi.org/10.1002/rmv.1974

56. Sykes L, MacIntyre DA, Yap XJ, Teoh TG, Bennett PR (2012) The Th1:Th2 dichotomy of pregnancy and preterm labour. Mediat Inflamm 2012:967629-967612. https://doi.org/10.1155/2012/ 967629

57. Scott GM, Chow SS, Craig ME et al (2012) Cytomegalovirus infection during pregnancy with maternofetal transmission induces a proinflammatory cytokine bias in placenta and amniotic fluid. J Infect Dis 205(8):1305-1310. https://doi.org/10.1093/infdis/jis 186

58. Takasu N, Ikema T, Komiya I, Mimura G (2005) Forty-year observation of 280 Japanese patients with congenital rubella syndrome. Diabetes Care 28(9):2331-2332. https://doi.org/10.2337/diacare. 28.9.2331

59. Op de Beeck A, Eizirik DL (2016) Viral infections in type 1 diabetes mellitus — why the beta cells? Nat Rev Endocrinol 12(5):263273. https://doi.org/10.1038/nrendo.2016.30

60. Nejentsev S, Walker N, Riches D, Egholm M, Todd JA (2009) Rare variants of IFIH1, a gene implicated in antiviral responses, protect against type 1 diabetes. Science 324(5925):387-389. https://doi. org/10.1126/science.1167728

61. Clausen TD, Bergholt T, Bouaziz O et al (2016) Broad-spectrum antibiotic treatment and subsequent childhood type 1 diabetes: a nationwide Danish cohort study. PLoS One 11(8):e0161654. https://doi.org/10.1371/journal.pone.0161654

62. Morgan NG, Richardson SJ (2018) Fifty years of pancreatic islet pathology in human type 1 diabetes: insights gained and progress made. Diabetologia 61(12):2499-2506. https://doi.org/10.1007/ s00125-018-4731-y

63. Dunne JL, Richardson SJ, Atkinson MA et al (2019) Rationale for enteroviral vaccination and antiviral therapies in human type $1 \mathrm{di}-$ abetes. Diabetologia 62(5):744-753. https://doi.org/10.1007/ s00125-019-4811-7

64. Pak CY, Eun HM, McArthur RG, Yoon JW (1988) Association of cytomegalovirus infection with autoimmune type 1 diabetes. Lancet 2(8601):1-4. https://doi.org/10.1016/s0140-6736(88) 92941-8

65. Bian X, Wallstrom G, Davis A et al (2016) Immunoproteomic profiling of antiviral antibodies in new-onset type 1 diabetes using protein arrays. Diabetes 65(1):285-296. https://doi.org/10.2337/ db15-0179

66. Ramondetti F, Sacco S, Comelli M et al (2012) Type 1 diabetes and measles, mumps and rubella childhood infections within the Italian Insulin-dependent Diabetes Registry. Diabet Med 29(6):761-766. https://doi.org/10.1111/j.1464-5491.2011.03529.x

67. Kolehmainen P, Koskiniemi M, Oikarinen S et al (2013) Human parechovirus and the risk of type 1 diabetes. J Med Virol 85(9): 1619-1623. https://doi.org/10.1002/jmv.23659

68. Yeung G, Rawlinson WD, Craig ME (2011) Enterovirus infection and type 1 diabetes mellitus: systematic review and meta-analysis 
of observational molecular studies. BMJ 342:d35. https://doi.org/ 10.1136/bmj.d35

69. Lonnrot M, Salminen K, Knip M et al (2000) Enterovirus RNA in serum is a risk factor for beta-cell autoimmunity and clinical type 1 diabetes: a prospective study. Childhood Diabetes in Finland (DiMe) Study Group. J Med Virol 61(2):214-220. https://doi.org/ 10.1002/(SICI)1096-9071(200006)61:2<214::AID-JMV7>3.0. $\mathrm{CO} ; 2-9$

70. Laitinen $\mathrm{OH}$, Honkanen H, Pakkanen O et al (2014) Coxsackievirus B1 is associated with induction of beta-cell autoimmunity that portends type 1 diabetes. Diabetes 63(2):446-455. https://doi.org/10.2337/db13-0619

71. Oikarinen S, Martiskainen M, Tauriainen S et al (2011) Enterovirus RNA in blood is linked to the development of type 1 diabetes. Diabetes 60(1):2769-2279. https://doi.org/10.2337/db10-0186

72. Perrett KP, Jachno K, Nolan TM, Harrison LC (2019) Association of rotavirus vaccination with the incidence of type 1 diabetes in children. JAMA Pediatr 173(3):280-282. https://doi.org/10.1001/ jamapediatrics.2018.4578

73. Rogers MAM, Basu T, Kim C (2019) Lower incidence rate of type 1 diabetes after receipt of the rotavirus vaccine in the United States, 2001-2017. Sci Rep 9(1):7727. https://doi.org/10.1038/s41598019-44193-4

74. Briese T, Kapoor A, Mishra N et al (2015) Virome capture sequencing enables sensitive viral diagnosis and comprehensive virome analysis. MBio 6(5):e01491-e01415. https://doi.org/10.1128/ mBio.01491-15

75. Kostic AD, Gevers D, Siljander H et al (2015) The dynamics of the human infant gut microbiome in development and in progression toward type 1 diabetes. Cell Host Microbe 17(2):260-273. https:// doi.org/10.1016/j.chom.2015.01.001

76. Koren O, Goodrich JK, Cullender TC et al (2012) Host remodeling of the gut microbiome and metabolic changes during pregnancy. Cell 150(3):470-480. https://doi.org/10.1016/j.cell.2012.07.008

77. DiGiulio DB, Callahan BJ, McMurdie PJ et al (2015) Temporal and spatial variation of the human microbiota during pregnancy. Proc Natl Acad Sci U S A 112(35):11060-11065. https://doi.org/10. 1073/pnas. 1502875112

78. Haupt-Jorgensen M, Morgen CS, Jess T et al (2018) Maternal antibiotic use during pregnancy and type 1 diabetes in children-a national prospective cohort study. Diabetes Care 41(12):e155e157. https://doi.org/10.2337/dc18-1764

79. Arpaia N, Campbell C, Fan X et al (2013) Metabolites produced by commensal bacteria promote peripheral regulatory T-cell generation. Nature 504(7480):451-455. https://doi.org/10.1038/ nature 12726

80. Gavin PG, Mullaney JA, Loo D et al (2018) Intestinal metaproteomics reveals host-microbiota interactions in subjects at risk for type 1 diabetes. Diabetes Care 41(10):2178-2186. https:// doi.org/10.2337/dc18-0777

81. Marino E, Richards JL, McLeod KH et al (2017) Gut microbial metabolites limit the frequency of autoimmune $\mathrm{T}$ cells and protect against type 1 diabetes. Nat Immunol 18(5):552-562. https://doi. org/10.1038/ni.3713

82. de Goffau MC, Fuentes S, van den Bogert B et al (2014) Aberrant gut microbiota composition at the onset of type 1 diabetes in young children. Diabetologia 57(8):1569-1577. https://doi.org/10.1007/ s00125-014-3274-0

83. Eizirik DL, Miani M, Cardozo AK (2013) Signalling danger: endoplasmic reticulum stress and the unfolded protein response in pancreatic islet inflammation. Diabetologia 56(2):234-241. https://doi.org/10.1007/s00125-012-2762-3

84. Thomaidou S, Zaldumbide A, Roep BO (2018) Islet stress, degradation and autoimmunity. Diabetes Obes Metab 20(Suppl 2):88 94. https://doi.org/10.1111/dom.13387

85. Kracht MJ, van Lummel M, Nikolic T et al (2017) Autoimmunity against a defective ribosomal insulin gene product in type 1 diabetes. Nat Med 23(4):501-507. https://doi.org/10.1038/nm.4289

86. Kaplan RM, Chambers DA, Glasgow RE (2014) Big data and large sample size: a cautionary note on the potential for bias. Clin Transl Sci 7(4):342-346. https://doi.org/10.1111/cts.12178

87. Mayer-Davis EJ, Kahkoska AR, Jefferies C et al (2018) ISPAD Clinical Practice Consensus Guidelines 2018: definition, epidemiology and classification of diabetes in children and adolescents. Pediatr Diabetes 19(Suppl 27):7-19. https://doi.org/10.1111/pedi. 12773

Publisher's note Springer Nature remains neutral with regard to jurisdictional claims in published maps and institutional affiliations. 